\title{
CONFIABILIDADE INTRA E INTEREXAMINADORES DE DOIS MÉTODOS DE MEDIDA DA AMPLITUDE ATIVA DE DORSIFLEXÃO DO TORNOZELO EM INDIVÍDUOS SAUDÁVEIS
}

\author{
Venturini $\mathrm{C}^{1}$, Ituassú NT ${ }^{2}$, Teixeira LM ${ }^{2}$ e Deus CVO ${ }^{2}$ \\ ${ }^{1}$ Departamento de Fisioterapia, Centro Universitário de Belo Horizonte, Belo Horizonte, MG; Departamento de \\ Fisioterapia, Pontifícia Universidade Católica de Minas Gerais, Betim, MG - Brasil \\ ${ }^{2}$ Departamento de Fisioterapia, Escola de Educação Física, Fisioterapia e Terapia Ocupacional, Universidade Federal \\ de Minas Gerais, Belo Horizonte, MG - Brasil \\ Correspondência para: Claudia Venturini, Rua Jornalista Guilherme Apgaua, 96/201, Buritis, CEP 30575-270, Belo \\ Horizonte, MG - Brasil,e-mail: clavent@terra.com.br
}

Recebido: 30/08/2005 - Revisado: 10/05/2006 - Aceito: 03/08/2006

\begin{abstract}
RESUMO
Introdução: A medida da amplitude do movimento (ADM) é um importante parâmetro utilizado na avaliação e no acompanhamento fisioterapêutico, conseqüentemente, a confiabilidade dessa medida e dos instrumentos utilizados devem ser avaliados. Objetivos: O objetivo deste estudo foi avaliar a confiabilidade das medidas intra-examinador e interexaminador da ADM ativa de dorsiflexão do tornozelo, por meio da goniometria e de forma mais funcional em cadeia cinética fechada (CCF). Materiais e métodos: Dois examinadores realizaram, em dois dias de teste, as mensurações de ambos os membros de 22 sujeitos saudáveis. A ADM ativa de dorsiflexão foi medida primeiro com o sujeito em prono, utilizando o goniômetro universal e, posteriormente, com o sujeito em dorsiflexão, na posição ortostática com o pé testado sobre uma fita métrica. O coeficiente de correlação intraclasse (CCI) foi utilizado para a análise da confiabilidade das medidas, e o teste t pareado e independente foi utilizado para verificar a diferença entre as médias de dois dias de teste e entre os dois examinadores, respectivamente. Resultados: Os coeficientes de correlação intraclasse (CCI) demonstraram de baixa a moderada confiabilidade intra-examinador, com CCI: 0,32 a 0,72, e moderada confiabilidade interexaminador, com CCI de 0,57 e 0,66 para a goniometria. Para a medida em CCF a confiabilidade foi alta tanto para a condição intra-examinador (CCI de 0,93 e 0,96 para os tornozelos direito e esquerdo, respectivamente) quanto para interexaminador (CCI de 0,98 e 0,99 para os tornozelos direito e esquerdo, respectivamente). Conclusão: Esses resultados indicaram que a confiabilidade da avaliação em CCF é maior que a do goniômetro universal, e isso indica ser um método confiável para sua aplicação clínica ao envolver o mesmo ou diferentes avaliadores.
\end{abstract}

Palavras-chave: amplitude de movimento, goniometria, tornozelo, avaliação funcional.

\section{ABSTRACT \\ Intrarater and Interrater Reliability of Two Methods for Measuring the Active Range of Motion for Ankle Dorsiflexion in Healthy Subjects}

Background: Range of motion (ROM) measurements are an important parameter for physiotherapeutic assessment and follow-up. Consequently, the reliability of such measurements and the instruments utilized must be evaluated. Objetive: To evaluate the intrarater and interrater reliability of active ROM measurements for ankle dorsiflexion using a goniometer and the more functional method of closed kinetic chains (CKC). Method: Two examiners measured both ankles of 22 healthy subjects, on two test days. The active ROM for dorsiflexion was first measured with the subject in the prone position using a universal goniometer and subsequently with the subject in the orthostatic position, with the foot to be tested in dorsiflexion on a measuring tape. The intraclass correlation coefficient (ICC) was used to analyze the reliability of the measurements, and Student's $\mathrm{t}$ test for paired and independent samples was used to investigate differences between the means for the two test days and between the two examiners, respectively. Results: The ICC showed low to moderate intrarater reliability (ICC: 0.32-0.72) and moderate interrater reliability (ICC: 0.57-0.66) for the goniometer measurements. For the CKC measurements, both intrarater reliability and interrater reliability were high: intrarater ICC of 0.93 and 0.96 for the right and left ankles, respectively; interrater ICC of 0.98 and 0.99 for the right and left ankles, respectively. Conclusion: These results indicated that the reliability of the CKC evaluation was greater than the reliability of the universal goniometer. This shows that CKC is a reliable method for clinical application involving the same or different examiners.

Key words: Range of motion, goniometry, ankle, functional test. 


\section{INTRODUÇÃO}

A medida da amplitude de movimento é parâmetro determinante utilizado na avaliação e no acompanhamento fisioterapêutico. Muitas vezes, a avaliação da amplitude de movimento faz parte da definição da propedêutica e do prognóstico de um indivíduo submetido à fisioterapia ${ }^{1,2}$.

O tornozelo é a articulação mais lesada do sistema musculoesquelético. A principal disfunção dessa articulação é o entorse lateral do tornozelo, que acomete um em cada 10.000 indivíduos no mundo inteiro e corresponde a 80\% das disfunções na articulação do tornozelo ${ }^{3,4}$.

O movimento de dorsiflexão do tornozelo é necessário para o desempenho funcional, principalmente para a marcha. Considera-se que cerca de $10^{\circ}$ desse movimento é necessário durante a fase de apoio médio da marcha ${ }^{5}$.

O método de mensuração da amplitude de movimento (ADM) mais utilizado na prática clínica é a goniometria ${ }^{6}$. Existem diferentes instrumentos para avaliar essas medidas como o goniômetro fluido, o eletrogoniômetro e o goniômetro universal ${ }^{7}$. O goniômetro universal é de fácil aplicação, não invasivo, de baixo custo e, por isso, o mais utilizado na clínica fisioterapêutica. Por outro lado, a reprodutibilidade de suas medidas é mais limitada quando comparada a outros goniômetros, principalmente quando envolve diferentes examinadores, o que limita as reavaliações periódicas que envolvam essas condições ${ }^{8}$. Além disso, a reprodutibilidade do goniômetro universal é examinador-dependente e varia de acordo com o nível de treinamento ${ }^{1}$.

A confiabilidade das medidas demonstra sua consistência obtida por um instrumento ou por um examinador nas mesmas condições de avaliação. A confiabilidade intra-examinador é a consistência das medidas realizadas nas mesmas condições de avaliação em dois momentos diferentes. Já a confiabilidade interexaminador vincula-se à consistência das medidas realizadas por dois examinadores diferentes ${ }^{1}$.

A padronização da metodologia é parâmetro fundamental para controlar as fontes de erro, proporcionando, assim, uma medida confiável ${ }^{9}$. Não existe consenso na literatura quanto à padronização da medida da articulação do tornozelo ${ }^{10}$. A posição de teste, o procedimento das medidas e os pontos de referência variam na bibliografia específica9 ${ }^{9}$

A goniometria de dorsiflexão do tornozelo apresenta boa confiabilidade intra-examinador e baixa confiabilidade interexaminador ${ }^{7,9}$. Diante desse fato, alguns autores propuseram outras formas de mensuração da dorsiflexão do tornozelo. Bennel et $\mathrm{al}^{11}$ sugeriram uma mensuração funcional, em cadeia cinética fechada, para a avaliação da dorsiflexão do tornozelo: enquanto o movimento é realizado próximo a uma parede, a dorsiflexão é mensurada indiretamente através da uma fita métrica atada ao chão. A distância entre o $1^{\circ}$ dedo do pé e a parede é considerada o resultado do teste e mensurada em centímetros. Trata-se de um método simples e confiável tanto para a condição intra-examinador quanto interexaminador. Contudo, a sua aplicabilidade é limitada aos indivíduos que podem realizar descarga de peso sobre o membro inferior ${ }^{11}$.

O desfecho da abordagem fisioterapêutica depende de mensurações padronizadas e confiáveis. Dessa forma, o objetivo do presente estudo foi avaliar a confiabilidade intra-examinador e interexaminador da goniometria e da mensuração em cadeia cinética fechada do movimento de dorsiflexão do tornozelo.

\section{MATERIAIS E MÉTODOS}

O estudo foi desenvolvido após a aprovação do Comitê de Ética em Pesquisa e todos os voluntários assinaram o termo de consentimento livre e esclarecido.

Dois examinadores, estudantes do curso de pósgraduação da UFMG, realizaram um treinamento prévio por um período de uma semana.

\section{Voluntários}

Foram recrutados 22 voluntários ( 20 mulheres e 2 homens), saudáveis, estudantes de cursos de fisioterapia, com idades entre 21 e 26 anos. Os critérios de exclusão foram presença de dor ou qualquer disfunção musculoesquelética ou neurológica nos últimos 6 meses que atingisse a articulação do tornozelo.

\section{Procedimentos}

Inicialmente, os voluntários receberam as informações quanto aos objetivos do estudo e foram familiarizados com o procedimento e o movimento de dorsiflexão que seria requerido durante os dois testes.

Em seguida, os voluntários foram posicionados em decúbito ventral, com o pé posicionado para fora da mesa, de acordo com a metodologia proposta por Jonson e Gross ${ }^{12}$ para a goniometria de dorsiflexão do tornozelo. O membro inferior oposto foi posicionado em abdução, rotação externa e flexão do joelho para corrigir a rotação tibial da perna. $\mathrm{O}$ primeiro examinador (A) marcava com lápis dermográfico os pontos de referência anatômica: cabeça do quinto metatarsal e linha que divide ao meio a fibula. Em seguida, a linha mediana do braço fixo do goniômetro foi posicionado sobre a fíbula e a linha externa do braço móvel sobre a cabeça do quinto metatarsal. O fulcro do goniômetro foi secundariamente posicionado abaixo do maléolo lateral, o que corresponde ao eixo da articulação. O tornozelo foi colocado em posição neutra e, em seguida, o voluntário foi orientado a realizar o movimento de dorsiflexão ativa do tornozelo, de acordo com a Figura 1. As mensurações foram repetidas três vezes consecutivas para cada tornozelo e a leitura foi realizada pelo próprio examinador. Em seguida, o segundo examinador (B) repetiu os mesmos procedimentos. As marcas de lápis dermográfico foram apagadas com álcool para não influenciar a medição feita pelo segundo examinador. 


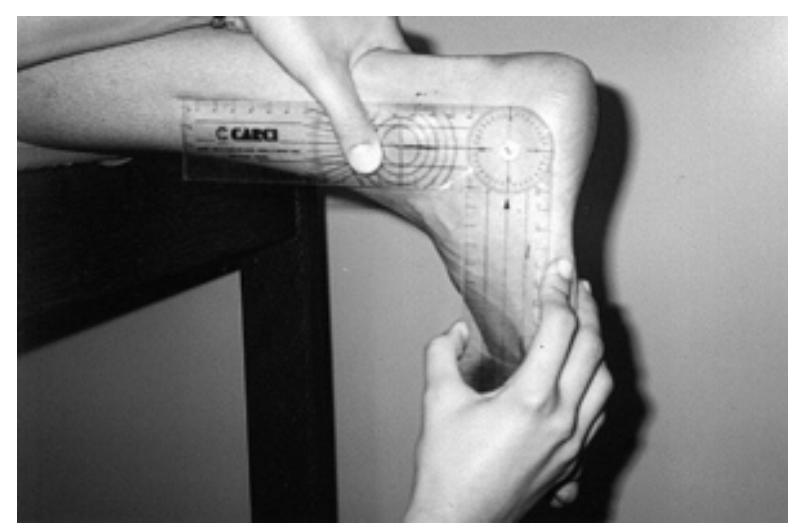

Figura 1. Goniometria de dorsiflexão do tornozelo.

Após a goniometria, os voluntários foram submetidos à mensuração da dorsiflexão em cadeia cinética fechada (CCF). Para isso, os voluntários foram posicionados em ortostatismo, com o pé a ser avaliado sobre uma fita métrica que se encontrava atada ao chão, em linha reta, a partir de uma parede. O hálux permanecia sobre a fita, enquanto o joelho fletido encostava na parede. O voluntário foi orientado a realizar o movimento de dorsiflexão em CCF, deslizando o pé na direção posterior, até o máximo possível, sem retirar o joelho da parede e o calcanhar do chão, de acordo com a Figura 2. Quando a dorsiflexão máxima foi atingida pelo voluntário, o examinador fez a leitura da distância entre o hálux e a parede, de acordo com os procedimentos propostos por Bennel et $\mathrm{al}^{11}$. Os mesmos procedimentos foram realizados tanto pelo examinador A quanto pelo examinador B.

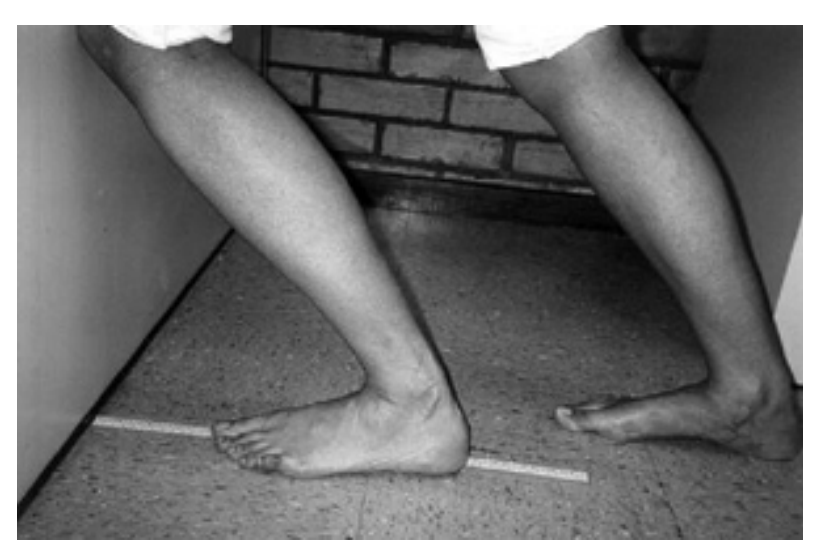

Figura 2. Medida de dorsiflexão em CCF.

As mensurações foram realizadas por dois examinadores nas mesmas condições de avaliação. Para a avaliação da confiabilidade intra-examinador, foi dado um intervalo de 48 horas entre os dois testes de cada examinador. Um terceiro examinador foi responsável pelo registro dos dados em formulários independentes para evitar, assim, a comparação entre os dados durante a coleta dos mesmos.

Os voluntários foram instruídos a não realizarem aquecimento ou qualquer atividade física 2 horas antes do horário previsto para a coleta de dados, a fim de evitar adaptação viscoelástica dos músculos envolvidos no estudo.

\section{Análise Estatística}

Feita uma análise descritiva dos dados, a confiabilidade da ADM de dorsiflexão foi determinada através do cálculo do coeficiente de correlação intraclasse $(\mathrm{CCI})^{1}$. O teste $\mathrm{t}$ pareado e o teste $t$ independente foram utilizados para avaliar a diferença entre os valores médios dos dois testes com intervalo de 48 horas e entre os valores médios dos dois examinadores, respectivamente. Os procedimentos estatísticos foram realizados utilizando o programa SPSS (Statistical Pakage for Science Social), versão 10.1. A confiabilidade foi considerada pequena (até 0,25$)$, baixa $(0,26-0,49)$, moderada $(0,50-0,69)$, alta $(0,70-0,89)$ e muito alta (acima de 0,90$)$, de acordo com os valores de referência descritos por Domholdt Apud Jonson e Gross ${ }^{12}$.

\section{RESULTADOS}

Os valores médios da goniometria e da medida em CCF para os dois testes com intervalo de 48 horas estão apresentados na tabela. As médias das medidas obtidas para a condição intra-examinador não apresentaram diferença estatisticamente significativa tanto para a medida de goniometria quanto para a medida em CCF. Os resultados da análise da confiabilidade intra-examinador, com intervalo de 48 horas entre os testes, demonstraram de baixa a moderada confiabilidade para as medidas de goniometria e em CCF de acordo com a Tabela 1.

Os resultados da confiabilidade interexaminador demonstraram confiabilidade moderada para a goniometria universal, com CCI de 0,57 e 0,66 para os tornozelos direito e esquerdo, respectivamente. A análise da confiabilidade interexaminador para a medida em CCF demonstrou alta confiabilidade, com CCI de 0,98 e 0,99 para os tornozelos direito e esquerdo, respectivamente. Quando a diferença entre as médias das medidas dos examinadores para a goniometria foi verificada, obteve-se diferença estatisticamente significativa $(\alpha=0,01)$. Na avaliação da diferença das médias das medidas entre os examinadores para a condição interexaminador não foi encontrada diferença estatisticamente significativa.

\section{DISCUSSÃO}

Os resultados do presente estudo demonstraram de baixa a moderada confiabilidade para a condição intra-examinador das medidas de goniometria para ambos os examinadores. Esses resultados são similares aos encontrados por Jonson 
Tabela 1. Valores médios da goniometria e da medida de dorsiflexão em cadeia cinética fechada (CCF) e coeficiente de correlação intraclasse (CCI).

\begin{tabular}{ccccccc}
\hline & $\begin{array}{c}\text { Exam 1 } \\
\text { Teste 1 }\end{array}$ & $\begin{array}{c}\text { Exam 1 } \\
\text { Teste 2 }\end{array}$ & $\begin{array}{c}\text { CCI } \\
\text { Exam 1 }\end{array}$ & $\begin{array}{c}\text { Exam 2 } \\
\text { Teste 1 }\end{array}$ & $\begin{array}{c}\text { Exam 2 } \\
\text { Teste 2 }\end{array}$ & $\begin{array}{c}\text { CCI } \\
\text { Exam 2 }\end{array}$ \\
\hline Gonio D & $16,0^{\circ} \pm 4,3$ & $16,5^{\circ} \pm 3,2$ & 0,38 & $10,8^{\circ} \pm 2,5$ & $11,2^{\circ} \pm 2,7$ & 0,72 \\
Gonio E & $16,9^{\circ} \pm 3,2$ & $15,7^{\circ} \pm 2,7$ & 0,47 & $13,3^{\circ} \pm 3,5$ & $12,1^{\circ} \pm 2,7$ & 0,32 \\
CCF D & $11,3 \pm 2,7 \mathrm{~cm}$ & $11,2 \pm 2,4 \mathrm{~cm}$ & 0,96 & $12,8 \pm 2,4 \mathrm{~cm}$ & $13,8 \pm 2,3 \mathrm{~cm}$ & 0,96 \\
CCF E & $11,5 \pm 2,8 \mathrm{~cm}$ & $12,0 \pm 2,5 \mathrm{~cm}$ & 0,95 & $11,2 \pm 2,6 \mathrm{~cm}$ & $11,57 \pm 2,5 \mathrm{~cm}$ & 0,93
\end{tabular}

Gonio D/E: goniometria do movimento de dorsiflexão dos tornozelos direito e esquerdo; CCF D/E: medidas da amplitude de movimento em cadeia cinética fechada dos tornozelos direito e esquerdo em centímetros (cm); Exam 1/ 2: Examinador 1 e 2.

e Gross ${ }^{12}$, que também obtiveram confiabilidade moderada na condição intra-examinador, utilizando a mesma metodologia.

No presente estudo, a confiabilidade intra-examinador foi avaliada com um intervalo de 48 horas entre os dois testes. A baixa e moderada confiabilidade encontradas nessa condição - CCI de 0,32 a 0,72 -, pode estar relacionada ao amplo intervalo de tempo utilizado, ao passo que, na maioria dos estudos de confiabilidade intra-examinador, os testes foram realizados com um intervalo de 15 minutos entre as medidas ${ }^{6}$. Por outra óptica, o estudo de Alphons et al. ${ }^{13}$ encontrou boa confiabilidade intra-examinador com coeficiente de correlação de 0,63 para a condição intra-examinador. Porém, esses autores utilizaram 8 medidas realizadas pelo mesmo examinador dentro de um intervalo de 3 meses. O coeficiente de variação dessas medidas foi de $43,8 \%$, indicando alta variação das medidas. De acordo com Portney e Watkins ${ }^{1}$, a homogeneidade dos dados pode ser uma das razões para uma baixa correlação. No presente estudo, a confiabilidade intra-examinador apresentou baixa variação do erro entre as medidas obtidas, enquanto que o CCI encontrado foi baixo. Quando as medidas são homogêneas, o CCI pode apresentar um escore menor devido à ausência de diferença estatística verificada na análise de variância dos dados.

Contudo, outros possíveis erros podem ter interferido nos resultados do presente estudo, como possíveis adaptações viscoelásticas devido a atividades físicas realizadas pelos voluntários dentro a do intervalo de 48 horas dos testes, subjetividade da posição neutra do tornozelo, alteração do posicionamento do voluntário e o movimento da pele sobre as proeminências ósseas do tornozelo durante a mensuração da goniometria ${ }^{8,9,14,15}$. Além disso, o examinador 1, sempre realizou as medidas seguido pelo examinador 2. Essa ordem poderia ter interferido nos resultados encontrados, porém as medidas foram realizadas ativamente, o que possivelmente não gerou adaptação viscoelástica dos tecidos. Isso pode ser justificado pela média das medidas encontradas pelo examinador 2, menores que as encontradas pelo examinador 1 .

Sabe-se que as medidas de goniometria universal dependem da experiência e do treinamento dos examinadores ${ }^{8}$.
No presente estudo, os examinadores eram inexperientes nos procedimentos de medida e realizaram somente uma semana de treinamento; e isso justificaria a baixa confiabilidade encontrada ${ }^{1}$.

Já no estudo da confiabilidade interexaminador, os resultados demonstraram confiabilidade moderada. Esses resultados também estão de acordo com Jonson e Gross ${ }^{12}$ e Youdas et $\mathrm{al}^{16}$. De acordo com o estudo de Youdas et al. ${ }^{16}$, existe um erro considerável da goniometria quando dois ou mais examinadores são avaliados, sendo, portanto, indicado que o mesmo terapeuta acompanhe as medidas ao longo da abordagem fisioterapêutica.

Os resultados do estudo da confiabilidade do método em CCF para a medida da ADM de dorsiflexão ativa do tornozelo demonstraram maior confiabilidade quando comparados ao método de goniometria. Esses resultados podem ser devido à maior facilidade no posicionamento do voluntário, o que favorece a reprodução das medidas ${ }^{14}$.

Esses resultados estão de acordo com Bennel et al. ${ }^{11}$, que também encontraram alta confiabilidade intra-examinador e interexaminador, demonstrando, portanto, que o método em CCF é confiável quando realizado por um ou por mais examinadores, desde que exista padronização da posição do pé durante a reprodução da medida. Também Ekstrand et al ${ }^{14}$, demonstraram alta confiabilidade da medida de dorsiflexão em CCF, porém esses autores utilizaram um inclinômetro digital para a mensuração da ADM.

No cenário clínico, uma medida não pode ser considerada significativa se não é válida e confiável ${ }^{1}$. Os pacientes podem ser avaliados e reavaliados pelo mesmo ou por diferentes fisioterapeutas, portanto a confiabilidade da medida é um importante parâmetro para permitir a consistência dos dados ao longo da evolução dos pacientes e nos estudos científicos $^{1,16}$. Assim, de acordo com os resultados do presente estudo, pode-se inferir que a goniometria do movimento de dorsiflexão do tornozelo é uma medida que apresenta de baixa a moderada confiabilidade, sendo necessários novos estudos com metodologias mais bem delimitadas para verificar a aplicabilidade desse método ao longo do acompanhamento 
dos pacientes com disfunções do complexo do pé e tornozelo. Além disso, o método em CCF demonstrou alta confiabilidade interexaminador, podendo ser utilizado por diferentes examinadores ao longo da abordagem fisioterapêutica.

Embora a goniometria seja amplamente utilizada na prática clínica, poucos estudos verificaram a confiabilidade e a padronização das medidas para a articulação do tornozelo. Assim como a goniometria de dorsiflexão, a medida em CCF, que utiliza a distância do hálux até a parede, ainda tem sido pouco explorada nos estudos científicos. Portanto, tornase fundamental o desenvolvimento de estudos que verifiquem sua validade, confiabilidade e aplicabilidade clínica.

\section{CONCLUSÃO}

A medida da ADM ativa de dorsiflexão do tornozelo, utilizando o goniômetro universal, apresentou confiabilidade intra-examinador e interexaminador de baixa a moderada. Por outro lado, as medidas realizadas em CCF apresentaram alta confiabilidade intra-examinador e interexaminador, o que sugere que esse método é mais confiável para a aplicação clínica que envolva um ou mais examinadores durante o acompanhamento fisioterapêutico.

\section{REFERÊNCIAS BIBLIOGRÁFICAS}

1. Portney LG, Watkins MP. Reliability. In: Portney LG, Watkins MP, editores. Foundations of clinical research. $2^{\text {a }}$ ed. New Jersey : Prentice Hall Health; 2000. P. 61 -75.

2. Wilson RW, Gansneder BM. Measures of functional limitation as predictors of disablement in athletes with acute ankle sprains. J Orthop Sports Phys Ther. 2000; (30): 528-35.

3. Lynch SA, Renstrom AFH. The of acute lateral ankle ligament rupture in the athlete. Sports Med. 1999; (27): 61-71.

4. Denegar CR, Hertel J, Fonseca J. The effect of lateral ankle sprain on dorsiflexion range of motion, posterior talar glide, and joint laxity. J Orthop Sports Phys Ther. 2002; (32): 166-173.
5. Lindsjö U, Danckwardt LG, Sahlstedt B. Measurement of the motion range in the loaded ankle. Clin Orthop Relat Res. 1985; (199): 68-71.

6. Allinger TL, Engsberg JR. A method to determine the range of motion of the ankle joint complex, in vivo. J Biomech. 1993; 26(1): 69-76.

7. Gogia PP, Braatz JH, Rose SJ, Norton BJ. Reliability and validity of goniometric measurements at the knee. Phys Ther. 1987; 67(2): 192-95.

8. Rome KM, Cowieson F. A reliability study of the universal goniometer, fluid goniometer, and electrogoniometer for the measurement of ankle dorsiflexion. Foot Ankle Int. 1996; 17(1): 28-32.

9. Miller PJ. Assesment of joint motion. In: Rothstein JM. Measurement in Physical Therapy. New York: Churchill Livingstone, 1985, p.103-135

10. Elveru RA, Rothstein JM, Lamb RL. Goniometric reliability in a clinical setting. subtalar and ankle joint measurements. Phys Ther. 1988; 5(68): 672-77.

11. Bennel K, Richard T, Henry W, Wassana T, David K. Intra-rater an inter-rater reliability of weigth-bearing lunge measure of ankle dorsiflexion. Aust J Physiother. 1998; (44): 175-80.

12. Jhonson LC, Gross MT. Intraexaminer Reliability, Interexaminer Reliability, and Mean Values for Nine Lower extremity Skeletal Measures in Healthy Naval Midshipmen. J Orthop Sports Phys Ther. 1997; (25):253-263.

13. Alphons MPM, et al. Variability and reliability of joint measurements. Am J Sports Méd. 1990; (18): 58-63.

14. Ekstrand J, Wiktorsson M, Öberg B, Gillquist J. Lower extremity goniometric measurements: A study to determine their reliability. Arch Phys Med Rehabil. 1982; (63): 171-75.

15. Moseley AM, Crosbie J, Adams R. Normative data for passive ankle plantarflexion - dorsiflexion flexibility. Clin Biomech. 2001; (16): 514-21.

16. Youdas JW, Bogard CL, Suman VJ. Reliability of goniometric measurements and visual estimates of ankle joint active range of motion obtained in a clinical setting. Arch Phys Med Rehabil. 1993; 10(74): 1113-18. 